\title{
On the Differential Evolution for Vehicle Routing Problem
}

\author{
Pavel Krömer, Ajith Abraham, Václav Snášel \\ IT4Innovations \& Faculty of Electrical \\ Engineering and Computer Science, \\ VŠB Technical University of Ostrava, \\ Ostrava, Czech Republic \\ Email: \{pavel.kromer,vaclav.snasel\}@vsb.cz, \\ ajith.abraham@ieee.org
}

\author{
Eshetie Berhan, Daniel Kitaw \\ Addis Ababa Institute of Technology \\ School of Mechanical and Industrial Engineering, \\ Addis Ababa University, \\ Addis Ababa, Ethiopia \\ Email: \{eshetie_ethio,danielkitaw\}@yahoo.com
}

\begin{abstract}
Vehicle Routing Problem (VRP) is a well known NP-hard optimization problem with a number of real world applications and a variety of different versions. Due to its complexity, large instances of VRP are hard to solve using exact methods. Instead, various heuristic and meta-heuristic algorithms were used to find feasible VRP solutions. This work proposes a Differential Evolution for VRP that simultaneously looks for an optimal set of routes and minimizes the number of vehicles needed. The algorithm is used to solve Stochastic VRP with Real Simultaneous Pickup and Delivery based on realworld data obtained from Anbessa City Bus Service Enterprise (ACBSE), Addis Ababa, Ethiopia. Additionally, the algorithm is evaluated on several well known VRP instances.
\end{abstract}

Keywords-vehicle routing problem; pickup and delivery; differential evolution

\section{INTRODUCTION}

The problem of designing a minimum cost set of routes to serve a collection of customers with a fleet of vehicles, known as Vehicle Routing Problem, is an important challenge in the field of logistics, distribution and transportation [1], partly because transportation and distribution contribute approximately $20 \%$ to the total costs of a product [2]. VRP was originally defined by Dantzig et al. [3] under the title "Truck dispatching problem" with the objective to design optimum routing of a fleet of gasoline delivery trucks between a bulk terminal and a large number of service stations supplied by the terminal.

This work proposes and evaluates a new version of Differential Evolution for the VRP. The algorithm uses permutation as a model of a set of routes and random keys encoding as a representation of the permutation. Such a representation is suitable for real parameter optimization methods such as the Differential Evolution (DE). The algorithm is evaluated on a new VRP model with stochastic pickup and delivery based on real-world data describing bus services in Anbessa City Bus Service Enterprise (ACBSE), Addis Ababa, Ethiopia. The model, first proposed in [4], is called Stochastic VRP with Real Simultaneous Pickup and Delivery (SVRPSPD). Additionally, several well-known instances of VRP are solved by the proposed algorithm in order to asses the quality of solutions found by the DE.

\section{Vehicle Routing Problem}

The VRP can be formulated in terms of graph theory. The basic VRP is defined as: let $G=\left(V_{n}, A\right)$ be a directed or asymmetric graph where $V_{n}=\left\{v_{0}, \ldots v_{n}\right\}$ is a set of vertices representing cities or bus stops with depot located at vertex $v_{0}$, and $A$ is the set of arcs. With every arc $(i, j) \in A, i \neq j$ is associated a non-negative weight $c_{i j}$. The set of weights associated with all arcs can be expressed by a distance matrix $C=\left(c_{i j}\right)$. In certain context, $c_{i j}$ can be interpreted as a travel cost or as a travel time [5]. When $C$ is symmetrical, it is often convenient to replace $A$ by a set of undirected edges $E$ and reformulate the problem using symmetric undirected graph. In many practical cases, the cost or the distance matrix satisfies the triangular inequality such that $c_{i k}+c_{k j} \geq c_{i j}, \forall_{i, j, k} \in V$ [6]. The general or classical VRP consists of designing a set of at most $K$ delivery or collection routes such that each route starts and ends at the depot, each customer is visited exactly once by exactly one vehicle, the total demand of each route does not exceed the vehicle capacity and the total routing cost is minimized [6].

Because of the complexity and practical relevance of the VRP, significant research effort has been dedicated to the Bus Scheduling Problem (BSP) and its other variants and many optimization models have been proposed [7]. Many models have focused on obtaining near optimal VRP solutions at reasonable computational costs. [1]. Various extensions for the Vehicle Schedule Problem (VSP) or VRP with different additional requirements were also investigated in the last fifty years [6]. Among others, VRP with one depot [8] or more than one depot [9], VRP with heterogeneous fleet with multiple vehicle types [8], VRP with variable trip departure times, VRP with Stochastic Demand [1], [10], [11], and VRP with incapacitated vehicles [12], [11] were considered.

\section{DifFEREnTIAL Evolution}

The DE is a versatile and easy to use stochastic evolutionary optimization algorithm [13]. It is a population-based optimizer that evolves a population of real encoded vectors 
representing the solutions to given problem. The DE was introduced by Storn and Price in 1995 [14], [15] and it quickly became a popular alternative to the more traditional types of evolutionary algorithms. It evolves a population of candidate solutions by iterative modification of candidate solutions by the application of the differential mutation and crossover [13].

The DE starts with an initial population of $N$ real-valued vectors. The vectors are initialized with real values either randomly or so, that they are evenly spread over the problem space. The latter initialization leads to better results of the optimization [13].

During the optimization, the DE generates new vectors that are scaled perturbations of existing population vectors. The algorithm perturbs selected base vectors with the scaled difference of two (or more) other population vectors in order to produce the trial vectors. The trial vectors compete with members of the current population with the same index called the target vectors. If a trial vector represents a better solution than the corresponding target vector, it takes its place in the population [13].

There are two most significant parameters of the DE [13]. The scaling factor $F \in[0, \infty]$ controls the rate at which the population evolves and the crossover probability $C \in[0,1]$ determines the ratio of bits that are transferred to the trial vector from its opponent. The size of the population and the choice of operators are another important parameters of the optimization process.

The basic operations of the classic DE can be summarized using the following formulas [13]: the random initialization of the $i$ th vector with $N$ parameters is defined by

$$
x_{i}[j]=\operatorname{rand}\left(b_{j}^{L}, b_{j}^{U}\right), \quad j \in\{0, \ldots, N-1\}
$$

where $b_{j}^{L}$ is the lower bound of $j$ th parameter, $b_{j}^{U}$ is the upper bound of $j$ th parameter and $\operatorname{rand}(a, b)$ is a function generating a random number from the range $[a, b]$. A simple form of the differential mutation is given by

$$
v_{i}^{t}=v_{r 1}+F\left(v_{r 2}-v_{r 3}\right)
$$

where $F$ is the scaling factor and $v_{r 1}, v_{r 2}$ and $v_{r 3}$ are three random vectors from the population. The vector $v_{r 1}$ is the base vector, $v_{r 2}$ and $v_{r 3}$ are the difference vectors, and the $i$ th vector in the population is the target vector. It is required that $i \neq r 1 \neq r 2 \neq r 3$. The uniform crossover that combines the target vector with the trial vector is given by

$$
\begin{aligned}
l & =\operatorname{rand}(0, N-1) \\
v_{i}^{t}[m] & =\left\{\begin{array}{l}
v_{i}^{t}[m] \\
x_{i}[m]
\end{array} \text { if }(\operatorname{rand}(0,1)<C) \text { or } m=l\right.
\end{aligned}
$$

for each $m \in\{1, \ldots, N\}$. The uniform crossover replaces with probability $1-C$ the parameters in $v_{i}^{t}$ by the parameters from the target vector $x_{i}$.
There are also many other modifications to the classic DE. Mostly, they differ in the implementation of particular DE steps such as the initialization strategy, the vector selection, the type of differential mutation, the recombination operator, and control parameter selection and usage [13].

\section{A. Recent Applications of DE to the VRP}

Large VRP instances cannot be practically solved by exact methods due to the NP-hardness of the problem. Instead, various heuristic and meta-heuristic algorithms were employed to find approximate VRP solutions in reasonable time [16], [17]. A categorized bibliography of different meta-heuristic methods applied to different VRP variants can be found in [17]. The DE has proved to be an excellent method for both, continuous and discrete optimization problems. This section provides a short overview of recent applications of the DE to different variants of VRP in 2013 and 2012.

Hou and Hou [18] and Hou et al. [19] used in 2013 and 2012 a new discrete differential evolution algorithm for stochastic vehicle routing problems with simultaneous pickups and deliveries. The algorithm used natural (integer) encoding with the symbol 0 as sub-route separator and fitness function incorporating routing objective and constraints. Besides the traditional DE operators, new bitwise mutation was proposed. The algorithm also utilized an additional revise operator to eliminate illegal chromosomes that might have been created during the evolution. The experiments conducted by the authors have shown that the proposed algorithm delivers better solutions and converges faster than other DE-based and GA-based VRP solvers.

Küçükoğlu and Öztürk [20] used a variant of DE to find solutions to VRP with backhauls and time windows. The problem was formulated using mixed integer programming, solved by the DE and tested on several benchmarks. A short comparison of four discrete DE algorithms on capacitated VRP was presented in [21].

Liu et al. [22] used in 2012 a memetic differential evolution algorithm to solve vehicle routing problem with time windows. The algorithm used a real-valued source space and discrete solution space. A source vector was translated into an solution vector by modifications of the source vector (e.g. insertion of sub-route separator ' 0 ' in feasible locations) and optimized by three local search algorithms. The fitness of the best routing found by the local searches was called generalized fitness of the source vector. The experiments performed by the authors have shown that the proposed modifications improve the quality of solutions found by the $\mathrm{DE}$ and that the new algorithm is especially suitable for solving VRP instances with clustered locations.

$\mathrm{Xu}$ and Wen [23] used differential evolution for unidirectional logistics distribution vehicle routing problem with no time windows. The authors approached the task as an multi-objective optimization problem (although none of the traditional multi-objective DE variants was used) and 
established an encoding scheme that mapped the real-valued candidate vector to a routing of $k$ vehicles.

\section{Differential Evolution for Vehicle Routing PROBLEM}

The VRP can be seen as a combinatorial optimization problem. The goal of the optimization is to find a set of routes connecting selected locations (bus stops, customers) so that each location is visited by a vehicle exactly once, each route starts and terminates in a special location (depot), considered constraints are satisfied, and selected objective function is minimized. In this work we represent a set of routes as a permutation of considered locations (without the depot) and separate each sub-route by a special sub-route separator similarly as e.g. in [16].

The DE proposed in this work uses permutation-based VRP representation, automatically optimizes the number of vehicles when an upper bound is given, and avoids the creation of illegal candidate solutions.

\section{A. Encoding}

There is a variety of possible encoding schemes for modelling permutations for populational meta-heuristic algorithms [24]. The DE uses real-encoded candidate solutions so a modified version of the random key (RK) encoding [25] was chosen. An RK encoded permutation is represented as a string of real numbers (random keys), whose position changes after sorting correspond to the permutation gene. The advantage of RK encoding is that it is at a large extent prone to creation of illegal solutions in course of the artificial evolution (e.g. by the crossover operator in Genetic Algorithms). The drawbacks of the RK encoding include computational complexity as it is necessary to perform a sorting of the random keys every time the candidate solution is decoded. The RK encoding translates a discrete combinatorial optimization problem into a real-valued optimization problem with a larger continuous search space.

The routing of a maximum of $k$ buses for $n$ locations (without the depot) is encoded as $\mathbf{x}=$ $\left(x_{1}, x_{2}, \ldots x_{k+n-1}\right), x_{i} \in \mathbb{R}$. Routing $\mathcal{R}$ is from the encoded vector $\mathbf{x}$ created according to Algorithm 1. During the decoding process, $k-1$ largest values of $\mathbf{x}$ are interpreted as route separators. The remaining values are used as random keys and translated into permutation of $n$ locations $\pi$. The values of $\pi$ are split into $k$ routes, each of which starts in the depot and terminates in the depot. Empty routes can be created when the vector $\mathrm{x}$ contains two or more route separators next to each other. The set of non-empty routes defines the routing $\mathcal{R}$.

\section{B. Fitness function}

Fitness functions used in this work are based on covered distance, number of routes, and penalty for bus capacity

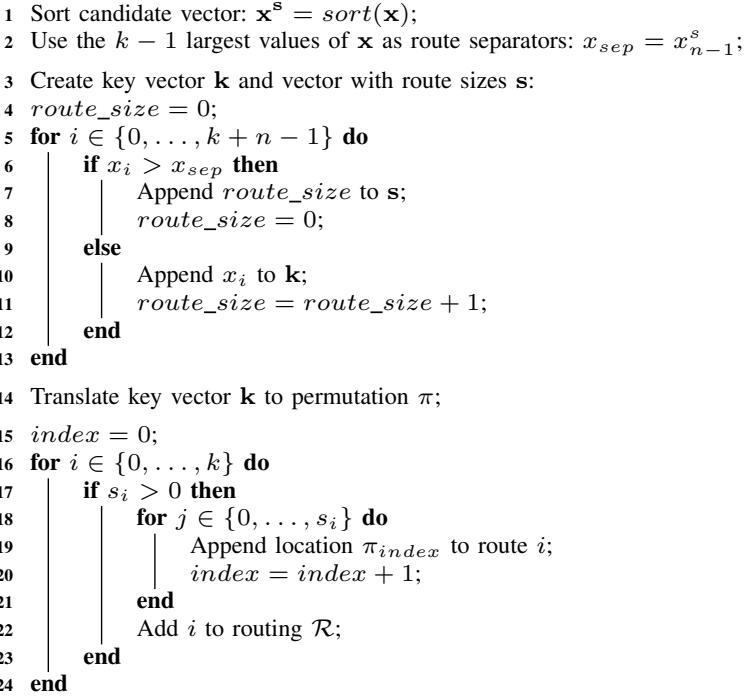

Algorithm 1: Decoding of routing $\mathcal{R}$

violation. Two variants of fitness function were considered:

$$
\begin{gathered}
f i t 1(\mathcal{R})=\frac{\sum_{r \in \mathcal{R}} \operatorname{dist}(r)}{|\mathcal{R}|} \\
f i t 2(\mathcal{R})=\left(\sum_{r \in \mathcal{R}} \operatorname{dist}(r)\right)|\mathcal{R}|
\end{gathered}
$$

where $\operatorname{dist}(r)$ is the distance of route $r$ and $|\mathcal{R}|$ represents the number of routes in $\mathcal{R}$. A penalty was applied (route distance was artificially increased) when the capacity of the bus was depleted.

\section{EXPERIMENTAL EVALUATION}

The DE was used to find solutions for different types of VRP instances. Moreover, the two fitness functions considered for the DE were evaluated and compared.

\section{A. Test Data}

The proposed DE was evaluated on a single instance of the Stochastic VRP with Real Simultaneous Pickup and Delivery based on the ANBESA data and on a set of VRP instances from the VRPH benchmark library [26].

1) SVRPSPD: The SVRPSPD model was first introduced in [4]. It considers simultaneous pickup and delivery at each bus stop. The pickup and delivery demand at each bus stop is treated as stochastic and random. The model also assumes that:

- The number of passengers to be picked up and dropped is random following a poisson probability distribution.

- The cumulative number of passengers picked up along the route must not exceed vehicle capacity $Q$.

- The fleet consists of homogeneous vehicles with limited capacity operating from a single depot. 
- Each vehicle can be used repeatedly within the planning horizon.

A VRP solution is considered feasible when the vehicles included in the solution served all the demand (for pick up and drop off) at each node along their paths without exceeding their capacity and violating additional constraints. The objective is to find a solution such that both the total traveled distance and the number of vehicles are minimized.

2) Capacitated VRP instances: In order to verify the proposed algorithm on a public set of VRP instances with known optimal solutions, the set of capacitated VRP (CVRP) instances (set $A$ ) by Augerat et al. [27] were downloaded from the VRPH website ${ }^{1}$ and solved by the DE. The data set consists of 27 VRP instances with different number of stops ranging from 32 to 80, fixed vehicle capacity, known optimum number of vehicles, and known best solution.

\section{B. VRP experiments}

The DE was implemented in $\mathrm{C}++$ and used to optimize routings of the ACBSE company and capacitated VRP instances from the VRPH website.

1) SVRPSPD experiments and results: The DE was executed with population size 100, maximum number of 20 vehicles, bus capacity of 70, 1000 generations, and parameters $F=0.9$ and $C=0.4$. The parameter values were set on the basis of initial experiments and algorithm tuning. The optimization was repeated 30 times due to the stochastic nature of the algorithm. Fitness function $f i t 1(\mathcal{R})$ was used.

The results of the optimization were: average number of routes 5.433, minimum number of routes 5 and maximum number of routes 7 . The fitness values in each generation of the 30 independent runs of the algorithm are shown in fig. 1. The results show that the algorithm is stable and

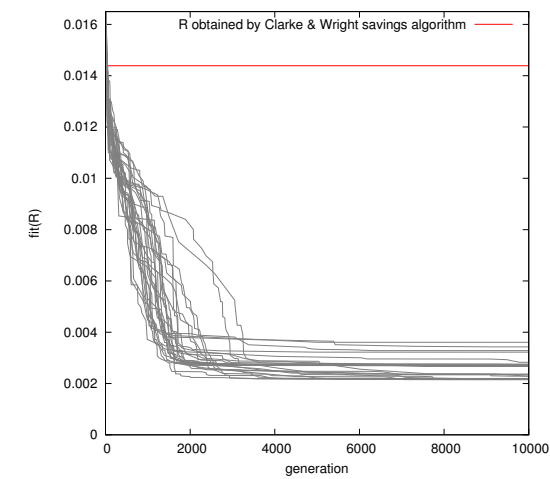

Figure 1: The evolution of $f i t 1(\mathcal{R})$ on SVRPSPD model.

able to reduce the estimated number of vehicles significantly. Moreover, the solutions found by the DE algorithm are better (in terms of used fitness function) than those found by a traditional savings algorithm by Clarke and Wrigth [28].

\footnotetext{
${ }^{1}$ https://sites.google.com/site/vrphlibrary/
}

2) Capacitated VRP experiments and results: The DE was executed with population size equal to dimension $\cdot 5$, maximum number of vehicles $\cdot 2$ vehicles, 25000 generations , and parameters $F=0.05$ and $C=1 e-6$. The parameter values were again set on the basis of initial experiments and the optimization was repeated 30 times. The results are shown in table I and a visual example of solutions of CVRP instances is provided in fig. 2. The visualisation was done using the vrp_plot tool by Groër et al. [26].

It can be seen that the errors of solutions found after 25000 generations by the DE with fitness function $\operatorname{fit} 1(\mathcal{R})$ were between 37.8 and $87.7 \%$. The errors of solutions obtained by DE with fitness function $f i t 2(\mathcal{R})$ were between 21.3 and $68.7 \%$. The latter fitness function was also more successful in reducing the number of vehicles. The evolution was at 25000th generation still ongoing and the quality of the solutions was improving.

\section{CONCLUSIONS}

This work proposed a DE for VRP and tested it on a recent VRP model (SVRPSPD) and well known set of CVRP instances. The DE encoded set of tours as a permutation and minimized the number of vehicles from an initial upper estimate. In contrast to a number of previous metaheuristic algorithms, all solutions generated by the proposed algorithm were valid routings and computational resources were not wasted on processing of invalid solutions.

The solution found by the algorithm for the SVRPSPD model was compared to a solution obtained by a traditional savings algorithm and it was found better with regard to used fitness function. The CVRP solutions obtained by the DE yielded quite large error when compared to known optimal solutions. On the other hand, the algorithm did not use any kind of local search or heuristic information in order to improve the results. Moreover, the computation was terminated before the DE converged and the results were still improving. The parameters of the DE algorithm could be also optimized to improve the results. Two fitness functions were tested and the second one, defined by eq. (6), was found better in terms of covered distance and number of vehicles of the solutions.

The results presented in this study are promising and meta-heuristic VRP solvers with various permutation-based representations of candidate solutions [24] will be investigated in the future.

\section{ACKNOWLEDGEMENT}

This work was supported by the European Regional Development Fund in the IT4Innovations Centre of Excellence project (CZ.1.05/1.1.00/02.0070) and by the Bio-Inspired Methods: research, development and knowledge transfer project, reg. no. CZ.1.07/2.3.00/20.0073 funded by Operational Programme Education for Competitiveness, co-financed by ESF and state budget of the Czech Republic. For the data used in this work, the researchers would like to also acknowledge ACBSE, Addis Ababa, Ethiopia. 


\begin{tabular}{l|ccccc}
\hline Instance & Best solution & $f i t 1(\mathcal{R})$ & $f i t 1(\mathcal{R})$ & $f i t 1$ error [\%] & fit2 error [\%] \\
\hline A-n32-k5 & 784 & 1174.28 & 1028.11 & 49.78061224 & 31.13647959 \\
A-n33-k5 & 661 & 910.89 & 837.362 & 37.80484115 & 26.68108926 \\
A-n33-k6 & 742 & 1122.98 & 967.589 & 51.34501348 & 30.40283019 \\
A-n34-k5 & 778 & 1120.68 & 998.161 & 44.04627249 & 28.29832905 \\
A-n36-k5 & 799 & 1148.12 & 1079.77 & 43.69461827 & 35.14017522 \\
A-n37-k5 & 669 & 1029.86 & 827.366 & 53.94020927 & 23.67204783 \\
A-n37-k6 & 949 & 1404.64 & 1174.25 & 48.01264489 & 23.73551106 \\
A-n38-k5 & 730 & 1135.72 & 920.877 & 55.57808219 & 26.14753425 \\
A-n39-k5 & 822 & 1176.2 & 1068.66 & 43.09002433 & 30.00729927 \\
A-n39-k6 & 831 & 1252.35 & 1237.46 & 50.70397112 & 48.91215403 \\
A-n44-k6 & 937 & 1375.59 & 1136.3 & 46.80789755 & 21.27001067 \\
A-n45-k6 & 944 & 1488.9 & 1306.95 & 57.72245763 & 38.44809322 \\
A-n45-k7 & 1146 & 1804.11 & 1523.22 & 57.42670157 & 32.91623037 \\
A-n46-k7 & 914 & 1538.43 & 1316.36 & 68.31838074 & 44.02188184 \\
A-n48-k7 & 1073 & 1609.48 & 1326.97 & 49.99813607 & 23.66915191 \\
A-n53-k7 & 1010 & 1667.92 & 1512.07 & 65.14059406 & 49.70990099 \\
A-n54-k7 & 1167 & 1822.56 & 1601.41 & 56.1748072 & 37.22450728 \\
A-n55-k9 & 1073 & 1799.49 & 1872.8 & 67.70643057 & 74.53867661 \\
A-n60-k9 & 1354 & 2099.25 & 2076.5 & 55.04062038 & 53.36041359 \\
A-n61-k9 & 1034 & 1695.63 & 1491.32 & 63.98742747 & 44.22823985 \\
A-n62-k8 & 1288 & 2144.33 & 2025.06 & 66.48524845 & 57.22515528 \\
A-n63-k10 & 1314 & 2114.7 & 2052.45 & 60.93607306 & 56.19863014 \\
A-n63-k9 & 1616 & 2698.48 & 2324.38 & 66.98514851 & 43.83539604 \\
A-n64-k9 & 1401 & 2243.46 & 2082.62 & 60.13276231 & 48.65239115 \\
A-n65-k9 & 1174 & 2198.69 & 1980.33 & 87.28194208 & 68.68228279 \\
A-n69-k9 & 1159 & 2025.44 & 1868.91 & 74.75754961 & 61.25194133 \\
A-n80-k10 & 1763 & 3308.55 & 2772.27 & 87.66591038 & 57.24730573 \\
\hline
\end{tabular}

Table I: Capacitated VRP solutions obtained by the DE.

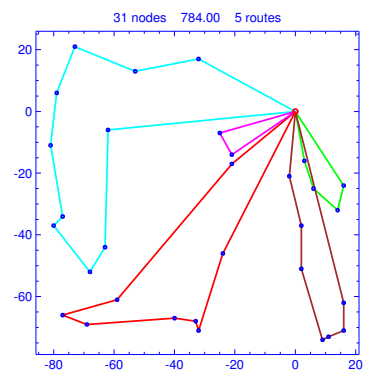

(a) A-n32-k5, best solution

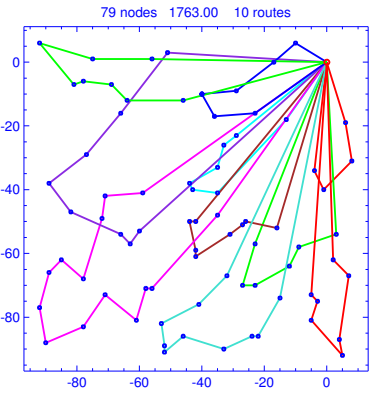

(d) A-n80-k10, best solution

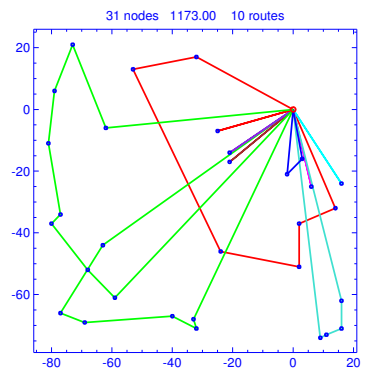

(b) A-n32-k5, fit $1(\mathcal{R})$

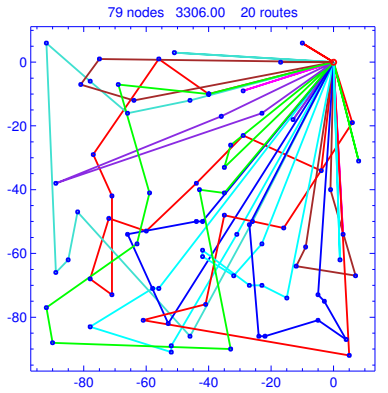

(e) A-n80-k10, $f i t 1(\mathcal{R})$

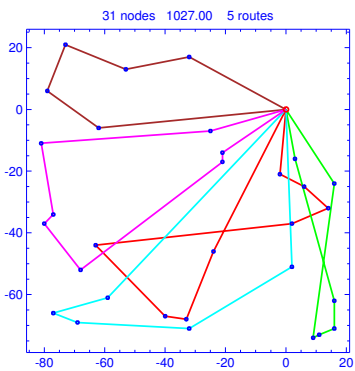

(c) A-n32-k5, fit2( $\mathcal{R})$

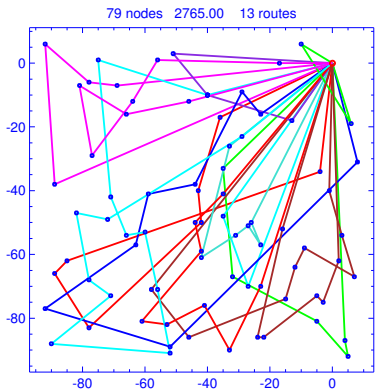

(f) A-n80-k10, fit2( $\mathcal{R})$

Figure 2: Visualization of A-n32-k5 and A-n80-k10 solutions.

This work was also partially supported by the Grant of SGS No. SP2013/70, VŠB - Tech. Univ. of Ostrava, Czech Republic.

\section{REFERENCES}

[1] J. Christopher, Goodson, "Solutions methodologies for vrp with stochastic demand," Dessirtation, Iowa, 2010. 
[2] M. Reimann, K. Doerner, and R. Hartl, "D-ants: Savings based ants divide and conquer the vehicle routing problem," Computers \& Operations Research, vol. 31, no. 4, pp. 563591, 2003.

[3] B. Dantzig, Gilbert and H. Ramser, J., "The truck dispatching problem," Journal of Management Science, Management Science, vol. 6, no. 1, pp. 80-91, 1959.

[4] E. Berhan, P. Krömer, D. Kitaw, A. Abraham, and V. Snášel, "Solving stochastic vehicle routing problem with real simultaneous pickup and delivery using differential evolution," in Innovations in Bio-inspired Computing and Applications, ser. AICS, A. Abraham, P. Krömer, and V. Snášel, Eds. Springer Int. Publishing, 2014, vol. 237, pp. 187-200.

[5] J.-F. Cordeau, G. Laporte, and A. Mercier, "A unifid tabu search heuristic for vehicle routing problem with time windows," J. of Operations Research Society, vol. 53, pp. 928936, 2001.

[6] P. Toth and D. Vigo, Eds., The Vehicle Routing Problem, ser. SIAM Monographs on Discrete Mathematics and Applications, Society for Industrial and Applied Mathematics. Philadelphia, PA, 19104-2688: Society for Industrial and Applied Mathematics, 2002.

[7] M. Dror and P. Trudeau, "Savings by split delivery routing," Transportation Science, vol. 23, pp. 141-145, 1989.

[8] H. I. Calvete, G. Carmen, O. María, José, and V. Belén, Sánchez, "Vehicle routing problems with soft time windows: an optimization based approach," J. of Monografías del Seminario Matemático García de Galdeano, vol. 31, pp. 295-304, 2004.

[9] D. Bertsimas, "A vehicle routing problem with stochastic demand," J. of Operations Research, vol. 40, no. 3, pp. 554585,1991

[10] N. Secomandi, "A rollout policy for the vehicle routing problem with stochastic demands," Operations Research, vol. 49, pp. 796-802, 2001.

[11] G. Laporte, F. Louveaux, and v. L. Hamme, "An integer 1shaped algorithm for the capacitated vehicle routing problem with stochastic demands," Operations Research, vol. 50, pp. 415-423, 2002.

[12] M. Gendreau, G. Laporte, and R. Seguin, "Stochastic vehicle routing," European J. of Operational Research, vol. 88, pp. 3-12, 1996a.

[13] K. V. Price, R. M. Storn, and J. A. Lampinen, Differential Evolution A Practical Approach to Global Optimization, Berlin, Germany: Springer-Verlag, 2005.

[14] R. Storn and K. Price, "Differential Evolution- A Simple and Efficient Adaptive Scheme for Global Optimization over Continuous Spaces,” Tech. Rep., 1995.

[15] R. Storn, "Differential evolution design of an IIR-filter," in Proceeding of the IEEE Conference on Evolutionary Computation ICEC. IEEE Press, 1996, pp. 268-273.

[16] E. Alba and B. Dorronsoro, "Solving the vehicle routing problem by using cellular genetic algorithms," in EvoCOP, ser. LNCS, J. Gottlieb and G. R. Raidl, Eds., vol. 3004. Springer, 2004, pp. 11-20.
[17] M. Gendreau, J.-Y. Potvin, O. Bräumlaysy, G. Hasle, and A. Løkketangen, "Metaheuristics for the vehicle routing problem and its extensions: A categorized bibliography," in The Vehicle Routing Problem: Latest Advances and New Challenges, ser. Oper. Research/Comp. Sci. Interfaces, B. Golden, S. Raghavan, and E. Wasil, Eds. Springer US, 2008, vol. 43, pp. 143-169.

[18] L. Hou and Z. Hou, "A novel discrete differential evolution algorithm for svrpspd," Applied Mechanics and Materials, vol. 303-306, pp. 2227-2230, 2013.

[19] L. Hou, Z. Hou, and H. Zhou, "Application of a novel discrete differential evolution algorithm to svrp," in Computational Sciences and Optimization (CSO), 2012 Fifth Int. Joint Conf. on, 2012, pp. 141-145.

[20] I. Küçükoğlu and N. Öztürk, "A differential evolution approach for the vehicle routing problem with backhauls and time windows," J. of Advanced Transportation, 2013 (in print)

[21] A. L. Silva, J. A. Ramírez, and F. Campelo, "A statistical study of discrete differential evolution approaches for the capacitated vehicle routing problem." in Proc. of the fifteenth annual conf. on Genetic and evolutionary computation companion, ser. GECCO '13 Companion. New York, NY, USA: ACM, 2013, pp. 77-78.

[22] W. Liu, X. Wang, and X. Li, "Memetic differential evolution for vehicle routing problem with time windows," in Advances in Swarm Intelligence, ser. LNCS, Y. Tan, Y. Shi, and Z. Ji, Eds. Springer Berlin Heidelberg, 2012, vol. 7331, pp. 358-365.

[23] H. Xu and J. Wen, "Differential evolution algorithm for the optimization of the vehicle routing problem in logistics," in Computational Intelligence and Security (CIS), 2012 Eighth Int. Conf. on, 2012, pp. 48-51.

[24] P. Krömer, J. Platoš, and V. Snášel, "Modeling permutations for genetic algorithms," in Proc. of the Int. Conf. of Soft Computing and Pattern Recognition (SoCPaR 2009). IEEE Computer Society, 2009, pp. 100 - 105.

[25] L. V. Snyder and M. S. Daskin, "A random-key genetic algorithm for the generalized traveling salesman problem," European J. of Operational Research, vol. 174, no. 1, pp. 38-53, 2006.

[26] C. Groër, B. Golden, and E. Wasil, "A library of local search heuristics for the vehicle routing problem," Mathematical Programming Computation, vol. 2, no. 2, pp. 79-101, 2010.

[27] P. Augerat, A. Corberan, E. Benavent, and J. Belenguer, "Computational results with a branch and cut code for the capacitated vehicle routing problem," Universite Grenoble 1. IMAG (Saint Martin d'Héres), Tech. Rep. RR 949-M, 1995.

[28] G. Clarke and J. Wright, "Scheduling of vehicles from a central depot to a number of delivery points," Operations Research, vol. 12, pp. 568-581, 1964. 